\title{
AVALIAÇÃO DO PROCESSO COMBINADO DE COAGULAÇÃO/FLOCULAÇÃO/FILTRAÇÃO REMOÇÃO DA MATÉRIA ORGÂNICA NATURAL DE ÁGUA DE ABASTECIMENTO UTILIZANDO COAGULANTE MORINGA OLEIFERA
}

T. R. T. Santos ${ }^{1 *}$, S. Bazana ${ }^{1}$, P. F. Coldebella ${ }^{1}$, F. S. Arakawa ${ }^{1}$, Q. L. Shimabuku ${ }^{1}$, C. Silveira $^{1}$, F. P. Camacho ${ }^{1}$, L. Nishi ${ }^{1}$, R. Bergamasco ${ }^{1}$, M. F. Vieira ${ }^{1}$

(1) Universidade Estadual de Maringá, Departamento de Engenharia Química E-mail para contato: tassia_tonial@hotmail.com

\begin{abstract}
RESUMO - O cloro utilizado na etapa desinfecção de águas pode reagir com a matéria orgânica natural (MON) proveniente dos mananciais e formar os trihalometanos (THM), que são tóxicos e carcinogênicos. Assim, este estudo propõe avaliar a eficiência do processo de coagulação/floculação $(\mathrm{C} / \mathrm{F})$ utilizando o coagulante policloreto de alumínio (PAC) em comparação ao coagulante natural sementes de Moringa oleifera sem óleo (extraído por hexano), Mo (hex), e esse associado ao polímero PA0823, Mo (hex) + pol, seguido por filtração para remoção da MON. Os ensaios foram realizados em Jar Test, utilizando água proveniente da bacia do rio Pirapó, Maringá, PR, com dosagens de coagulante de 9,5 mg. $\mathrm{L}^{-1}$ para o PAC, $30 \mathrm{mg} . \mathrm{L}^{-1}$ para Mo (hex) e para Mo (hex) + pol foi utilizada a dosagem ótima da Mo (hex) mais $0,1 \mathrm{mg} . \mathrm{L}^{-1}$ do polímero seguido de filtração em carvão ativado de coco de dendê. Após esses processos, o coagulante Mo (hex) + pol apresentou eficiências de remoção equivalentes ao coagulante sintético PAC.
\end{abstract}

\section{INTRODUÇÃO}

Os sistemas de abastecimento de água para consumo humano visam à garantia da potabilidade da água em todos os aspectos, de forma a eliminar os riscos de transmissão de agentes ou doenças de veiculação hídrica. Para cumprir com eficiência esta função é imprescindível um cuidadoso e adequado desenvolvimento de todas as fases de tratamento Brasil (2006).

Uma etapa importante no tratamento de água é a desinfecção, que tem como objetivo a destruição ou inativação da grande maioria dos organismos patogênicos, capazes de produzir doenças, ou de outros organismos indesejáveis. O cloro utilizado nessa etapa pode reagir com a matéria orgânica natural (MON) presente na água superficial formando subprodutos da cloração como os trihalometanos (THM), que são formados durante e após o tratamento, devido ao cloro residual obrigatoriamente presente na rede de distribuição, os quais são tóxicos e potencialmente carcinogênicos (Hong, 2007; Wang et al., 2012; Drinan et al., 2012). 
Assim, esse estudo propõe verificar a eficiência do processo coagulação/floculação (C/F), utilizando o coagulante natural Moringa oleífera (Mo) após a extração do óleo com hexano, combinado ao polímero PA 0823, com posterior filtração em carvão ativado e póscloração, avaliando a remoção da MON, assim, como a minimização da formação de THM.

\section{MATERIAIS E MÉTODOS}

Amostras de água superficial bruta $(\mathrm{AB})$ utilizadas foram provenientes do rio Pirapó, captada pela Companhia de Saneamento do Paraná (SANEPAR) de Maringá-PR. Essa água foi submetida ao processo de $\mathrm{C} / \mathrm{F}$, utilizando o coagulante químico policloreto de alumínio (PAC) e o coagulante natural Mo, juntamente com o polímero PA 0823.

\subsection{Preparo dos Coagulantes}

As sementes maduras de Mo utilizadas, foram provenientes da Universidade Federal de Sergipe (UFS) removidas da vagem seca e descascadas manualmente.

Extração do óleo da semente de Mo por hexano pelo método de Soxhlet - Mo (hex): Esse processo realizou-se por meio da pesagem de $10 \mathrm{~g}$ de sementes de Mo trituradas e secas em estufa. $\mathrm{O}$ pó foi transferido para um cartucho de extração e levado a um extrator contínuo soxhlet com hexano durante 8 horas. A trituração serviu para facilitar o processo de extração, pois garantiu uma maior superfície de contato com o solvente.

Em seguida, 1 grama de sementes foi adicionada a $100 \mathrm{~mL}$ de solução salina $(\mathrm{NaCl} 1 \mathrm{M})$ em liquidificador doméstico. A solução foi então processada durante 30 minutos e em seguida filtrada em filtro qualitativo, obtendo uma solução $1 \%$ de sementes de Mo. A dosagem utilizada foi de $30 \mathrm{mg} \cdot \mathrm{L}^{-1}$.

Mo hexano + Polímero aniônico Mo (hex) + pol: Foi utilizada a solução salina de Mo (hex) como coagulante principal em associação com polímero aniônico. A solução padrão do polímero foi preparada adicionando $1 \mathrm{~g}$ de polímero aniônico PA $0823 \mathrm{em} 100 \mathrm{~mL}$ de água destilada. A dosagem utilizada foi de $0,1 \mathrm{mg} . \mathrm{L}^{-1}$ do polímero e $30 \mathrm{mg} . \mathrm{L}^{-1}$ de Mo (hex).

Policloreto de Alumínio (PAC): Para a preparação da solução padrão do coagulante PAC, foi considerada uma concentração de $1 \% \mathrm{v} / \mathrm{v}$, ou seja, para cada $1 \mathrm{~mL}$ de PAC, completou-se para um volume total de $100 \mathrm{~mL}$ com água destilada. A dosagem utilizada foi de $9,5 \mathrm{mg} . \mathrm{L}^{-1}$

Os ensaios de C/F foram realizados em Jar Test em diferentes condições de operação, otimizadas por Kalibbala et al. (2009) e fornecidas pela SANEPAR. A Tabela 1 mostra as condições de operação utilizadas nos ensaios em Jar Test. 
Tabela 1 - Condições de operação do Jar Test

\begin{tabular}{|l|c|c|c|}
\hline Condições de operação & PAC & Mo (hex $)^{2}$ & Mo (hex) + pol $^{2}$ \\
\hline Gradiente de mistura rápida $\left(\mathrm{s}^{-1}\right)$ & 1,0 & 1,0 & 1,0 \\
\hline Tempo de mistura rápida $(\mathrm{min})$ & 110 & 150 & 150 \\
\hline Gradiente de mistura lenta $\left(\mathrm{s}^{-1}\right)$ & 15 & 30 & 30 \\
\hline Tempo de mistura lenta $(\mathrm{min})$ & 45 & 30 & 30 \\
\hline Tempo de sedimentação (min) & 15 & 60 & 15 \\
\hline
\end{tabular}

${ }^{1}$ SANEPAR (Companhia de Saneamento de Maringá)

${ }^{2}$ Kalibbala et al. (2009)

Na etapa posterior, a água clarificada passou por um processo de filtração, utilizando uma coluna contendo carvão ativado de coco de dendê. Por fim, foi realizado o processo de desinfecção (pós-cloração) com hipoclorito de sódio na dosagem de 1,5 mg..-1 ${ }^{-1}$, que é a utilizada pela SANEPAR. Após a cloração foi avaliada a formação de THM nessa água.

As análises de trihalometanos totais (THMT) foram realizadas por meio de cromatografia gasosa em coluna capilar (CG), com sistema de separação por purge and trap, e detecção e quantificação por espectrometria de massa (EM), de acordo com o Método 6200B, Standard Methods for the Examination of Water and Wastewater Apha (1995).

Em todas as etapas foram analisados os seguintes parâmetros: cor aparente (espectrofotômetro DR $2010 \mathrm{Hach}$ ), turbidez (turbidímetro 2100P Hach), compostos com absorção em $\mathrm{UV}_{254 \mathrm{~nm}}\left(\mathrm{UV}_{254 \mathrm{~nm}}\right)$ (espectrofotômetro DR 2010 Hach), carbono orgânico dissolvido (COD) (analisador de carbono orgânico total TOC-LCPH Shimadzu), THMT e cloro livre residual (somente na etapa de desinfecção).

Para as análises estatísticas utilizou-se o delineamento inteiramente casualisado (DIC). Foram realizados para comparação dos resultados análise de variância (ANOVA) e o teste de comparação de média, teste Tukey, com 95\% de confiança, para verificar as diferenças significativas das eficiências de remoção dos parâmetros avaliados através do programa estatístico Statística versão 8.0.

\section{RESULTADOS E DISCUSSÕES}

A caracterização da água bruta utilizada no presente estudo está apresentada na Tabela 2

Tabela 2 - Caracterização da água bruta

\begin{tabular}{|c|c|}
\hline Parâmetros & Valores \\
\hline Cor aparente $(\mathrm{uH})^{(1)}$ & 153 \\
\hline Turbidez $(\mathrm{NTU})$ & 49 \\
\hline $\mathrm{UV}_{254 \mathrm{~nm}}$ & 0,221 \\
\hline COD $\left(\mathrm{mg} . \mathrm{L}^{-1}\right)$ & 4,455 \\
\hline THMT $\left(\mu \mathrm{g} . \mathrm{L}^{-1}\right)$ & 4,45 \\
\hline
\end{tabular}

(1) unidade Hazen $=\left(\mathrm{mg}\right.$ Pt-Co. $\left.\mathrm{L}^{-1}\right)$ 
O processo de $\mathrm{C} / \mathrm{F}$ com posterior filtração (C/F/Filtro) realizado em escala de bancada utilizando o coagulante natural Mo, foi proposto com a finalidade de comparar seu desempenho com o do coagulante químico PAC.

\subsection{Coagulação/Floculação}

Na Tabela 3 são apresentados os resultados do parâmetro cor aparente, analisados no processo de $\mathrm{C} / \mathrm{F}$.

Tabela 3 - Análise do parâmetro de qualidade Cor aparente no processo de C/F

\begin{tabular}{|c|c|c|c|}
\hline \multicolumn{4}{|c|}{ Cor Aparente } \\
\hline Coagulante & Remoção (\%) & Residual (uH) & \multirow{2}{*}{ Portaria 2914/2011 } \\
\hline Mo (hex) & $53,43^{\mathrm{b}} \pm 1,45$ & 71 & \multirow{2}{*}{$15 \mathrm{uH}$} \\
\hline Mo (hex) + pol & $53,59^{\mathrm{b}} \pm 6,96$ & 71 & \\
\hline PAC & $94,93^{\mathrm{c}} \pm 2,63$ & 7 & \\
\hline
\end{tabular}

Resultados expressos em valores médios \pm desvio padrão. As médias seguidas por mesma letra não diferem estatisticamente entre si, pelo teste de Tukey a 5\% de nível de significância.

Em relação ao parâmetro cor, podemos observar que tanto a solução com Mo (hex) quanto Mo (hex) + pol utilizadas no processo de coagulação/floculação, obtiveram remoções em torno de 53\%, não havendo diferença estatistica entre as amostras. Também podemos observar a necessidade de um tratamento posterior quando utilizado o coagulante natural Mo, pois o mesmo não conseguiu atingir os parâmetros exigidos pela Portaria 2914/2011 somente com este tratamento. O PAC apresentou remoções de $95 \%$.

$\mathrm{Na}$ Tabela 4 são apresentados os resultados do parâmetro turbidez, analisados no processo de $\mathrm{C} / \mathrm{F}$.

Tabela 4 - Análise do parâmetro de qualidade Turbidez no processo de C/F

\begin{tabular}{|c|c|c|c|}
\hline \multicolumn{4}{|c|}{ Turbidez } \\
\hline Coagulante & Remoção (\%) & Residual (uH) & \multirow{2}{*}{ Portaria 2914/2011 } \\
\hline Mo (hex) & $77,16^{\mathrm{b}} \pm 1,90$ & 11,1 & 5TU \\
\hline Mo (hex) + pol & $77,30^{\mathrm{b}} \pm 3,56$ & 11,2 & \\
\hline PAC & $96,93^{\mathrm{c}} \pm 0,61$ & 1,5 & \\
\hline
\end{tabular}

Resultados expressos em valores médios \pm desvio padrão. As médias seguidas por mesma letra não diferem estatisticamente entre si, pelo teste de Tukey a 5\% de nível de significância.

De acordo com a Tabela 4 observamos que em relação ao parâmetro turbidez, com uma única etapa de $\mathrm{C} / \mathrm{F}$ foi possível alcançar $77 \%$ de remoção com os coagulantes Mo (hex) e Mo (hex) + pol, remoções essas bastante significativas, mas ainda necessitando de um tratamento subsequente para atingir as normas exigidas pela portaria. As duas formas de preparo dos coagulantes naturais não se diferenciaram estatisticamente mostrando dessa forma que não ha necessidade do uso do auxiliar de coagulação para remoção desse parâmetro. Com o coagulante comercial PAC foi possível alcançar uma remoção de $97 \%$.

$\mathrm{Na}$ Tabela 5 são apresentados os resultados do parâmetro compostos com absorção em 
$\mathrm{UV}_{254 \mathrm{~nm}}$ no processo de $\mathrm{C} / \mathrm{F}$

Tabela 5 - Análise do parâmetro de qualidade dos compostos com absorção em $U_{254 n m}$ no processo de $\mathrm{C} / \mathrm{F}$

\begin{tabular}{|c|c|c|}
\hline \multicolumn{3}{|c|}{ Compostos com absorção em UV } \\
\hline 254nm \\
\hline Coagulante & Remoção (\%) & Residual \\
\hline Mo (hex) & $65,05^{\mathrm{b}} \pm 2,60$ & 0,08 \\
\hline Mo (hex) + pol & $64,19^{\mathrm{b}} \pm 3,66$ & 0,08 \\
\hline PAC & $93,89^{\mathrm{c}} \pm 3,59$ & 0,01 \\
\hline
\end{tabular}

Resultados expressos em valores médios \pm desvio padrão. As médias seguidas por mesma letra não diferem estatisticamente entre si, pelo teste de Tukey a 5\% de nível de significância.

Pela Tabela 5 observamos que quando foi utilizado apenas o processo de $\mathrm{C} / \mathrm{F}$, remoções muito próximas do parâmetro $\mathrm{UV}_{254 \mathrm{~nm}}$ foram alcançadas com Mo (hex) e Mo (hex) + pol, 65\% e 64\%, respectivamente, não tendo diferenças estatística entre as duas formas de preparo do coagulante, podemos dessa forma observar o quão eficiente foi do coagulante natural Mo na remoção desse parâmetro e reafirmando que não é necessária a utilização do polímero como auxiliar de coagulação. Para o PAC foi possível atingir uma remoção de $94 \%$.

Na Tabela 6 são apresentados os resultados do parâmetro COD, analisados quando utilizados no processo de $\mathrm{C} / \mathrm{F}$.

Tabela 6 - Análise do parâmetro de qualidade COD no processo de C/F

\begin{tabular}{|c|c|c|}
\hline \multicolumn{3}{|c|}{ COD } \\
\hline Coagulante & Remoção (\%) & Residual \\
\hline Mo (hex) & $37,07^{\mathrm{c}} \pm 0,02$ & 0,29 \\
\hline Mo (hex) + pol & $36,13^{\mathrm{c}} \pm 0,70$ & 0,29 \\
\hline PAC & $40,64^{\mathrm{d}} \pm 0,96$ & 0,27 \\
\hline
\end{tabular}

Resultados expressos em valores médios \pm desvio padrão. As médias seguidas por mesma letra não diferem estatisticamente entre si, pelo teste de Tukey a 5\% de nível de significância.

Em relação aos resultados de COD, podemos observar que apenas com pré-tratamento de $\mathrm{C} / \mathrm{F}$ não foi possível obter reduções significativas tanto para os coagulantes naturais (Mo hex) e Mo (hex) + pol quanto para o coagulante sintético PAC , com remoções de 37\%, 36\% e $40 \%$ respectivamente. Logo, se fez necessário o uso de técnicas complementares para remoção dessa fração dissolvida. Em relação à estatística os dois coagulantes naturais utilizados não se diferiram estatisticamente.

\subsection{Filtração}

Na Tabela 7 são apresentados os resultados do parâmetro cor aparente para o processo combinado (C/F/Filtro). 
Tabela 7 - Análise do parâmetro de qualidade cor aparente no processo combinado C/F/Filtro

\begin{tabular}{|c|c|c|c|}
\hline \multicolumn{4}{|c|}{ Cor Aparente } \\
\hline Coagulante & Remoção (\%) & Residual (uH) & \multirow{2}{*}{1} \\
\hline Mo (hex) & $96,79^{\mathrm{b}} \pm 3,00$ & 2 & \multirow{2}{*}{$\mathrm{uH}$} \\
\hline Mo (hex) + pol & $97,98^{\mathrm{bc}} \pm 1,62$ & 1 & \\
\hline PAC & $99,47^{\mathrm{c}} \pm 0,73$ & 0 & \\
\hline
\end{tabular}

Resultados expressos em valores médios \pm desvio padrão. As médias seguidas por mesma letra não diferem estatisticamente entre si, pelo teste de Tukey a 5\% de nível de significância.

De acordo com a Tabela 7, observamos que remoções semelhantes e bastante significativas em torno de $97 \%$ foram alcançadas quando se utilizou os coagulantes naturais, mostrando a importância da implementação de um processo subsequente e principalmente a eficiência de remoção desse parâmetro quando se utilizou o filtro recheado com carvão de coco de dende. Os dois coagulantes naturais não se diferiram estatisticamente e com a adição do polímero como auxiliar de coagulação foi possível alcançar remoções proximas ao do coagulante sintético PAC que foi de $99 \%$.

$\mathrm{Na}$ Tabela 8 são apresentados os resultados do parâmetro turbidez no processo combinado (C/F/Filtro).

Tabela 8 - Análise do parâmetro de qualidade Turbidez no processo combinado C/F/Filtro

\begin{tabular}{|c|c|c|c|}
\hline \multicolumn{4}{|c|}{ Turbidez } \\
\hline Coagulante & Remoção (\%) & Residual (uH) & \multirow{2}{*}{ Portaria 2914/2011 } \\
\hline Mo (hex) & $96,48^{\mathrm{b}} \pm 0,97$ & 0,4 & \multirow{2}{*}{5 NTU } \\
\hline Mo (hex) + pol & $97,43^{\mathrm{b}} \pm 0,79$ & 0,3 & \\
\hline PAC & $97,43^{\mathrm{b}} \pm 0,79$ & 0,3 & \\
\hline
\end{tabular}

Resultados expressos em valores médios \pm desvio padrão. As médias seguidas por mesma letra não diferem estatisticamente entre si, pelo teste de Tukey a 5\% de nível de significância.

Em relação ao parametro turbidez, constatamos que o processo combinado (C/F/Filtro) apresentou uma melhora significativa nas eficiências de remoção tanto para Mo (hex) quanto para Mo (hex) + pol com remoções de 96\%, 97\% respectivamente. Os dois coagulantes naturais obtiveram resultados abaixo do limite exigido pela legislação e não diferiram-se estatisticamente.

Na Tabela 9 são apresentados os resultados do parâmetro compostos com absorção em $\mathrm{UV}_{254 \mathrm{~nm}}$ para o processo combinado (C/F/Filtro).

Tabela 9 - Análise do parâmetro de qualidade compostos com absorção em $U^{254 n m}$ no processo combinado $\mathrm{C} / \mathrm{F} /$ Filtro

\begin{tabular}{|c|c|c|}
\hline \multicolumn{3}{|c|}{ Compostos com absorção em UV $\mathbf{2 5 4 n m}$} \\
\hline Coagulante & Remoção (\%) & Residual \\
\hline Mo (hex) & $96,13^{\mathrm{b}} \pm 2,76$ & 0,003 \\
\hline Mo (hex) + pol & $95,57^{\mathrm{b}} \pm 3,65$ & 0,004 \\
\hline PAC & $100^{\mathrm{c}} \pm 0$ & 0 \\
\hline
\end{tabular}


Resultados expressos em valores médios \pm desvio padrão. As médias seguidas por mesma letra não diferem estatisticamente entre si, pelo teste de Tukey a 5\% de nível de significância.

Quanto ao parâmetro $\mathrm{UV}_{254 \mathrm{~nm}}$, o processo combinado utilizando o cogulante natural Mo (hex) apresentou a maior eficiência de remoção, 96 \%, quando comparado ao processo utilizando o polímero catiônico, Mo (hex) + pol, que apresentou remoção de $95 \%$. Apesar do processo utilizando Mo (hex) ter apresentado a melhor eficiência de remoção comparando estatisticamente os dois coagulantes naturais não apresentaram diferença estatistica sendo igualmente eficientes. Utilizando o coagulante PAC foi possivel alcançar remoção de $100 \%$ deste parâmetro de qualidade.

Na Tabela 10 são apresentados os resultados do parâmetro COD para o processo combinado (C/F/Filtro).

Tabela 10 - Análise do parâmetro de qualidade COD no processo combinado C/F/Filtro

\begin{tabular}{|c|c|c|}
\hline \multicolumn{3}{|c|}{ COD } \\
\hline Coagulante & Remoção (\%) & Residual \\
\hline Mo (hex) & $99,76^{\mathrm{b}} \pm 0,13$ & 0,001 \\
\hline Mo (hex) + pol & $99,77^{\mathrm{b}} \pm 0,14$ & 0,001 \\
\hline PAC & $99,77^{\mathrm{b}} \pm 0,12$ & 0,001 \\
\hline
\end{tabular}

Resultados expressos em valores médios \pm desvio padrão. As médias seguidas por mesma letra não diferem estatisticamente entre si, pelo teste de Tukey a 5\% de nível de significância.

$\mathrm{O}$ uso do processo combinado ( $\mathrm{C} / \mathrm{F} /$ Filtro) permitiu um aumento significativo das eficiências de remoção para todos coagulantes testados sendo possível atingir remoções de $99, \%$ de COD.

Observa-se que os residuais dos parâmetros avaliados utilizando os coagulantes naturais foram idênticos ao coagulante químico PAC mostrando-se um processo bastante promissor para ser utilizado em substituição ao coagulante comercial.

\subsection{Formação de THMT}

Na Tabela 11 são apresentadas as concentrações de THMT na água bruta, após o processo combinado (C/F/Filtro) e após a cloração em diferentes tempos de retenção $(0,5 ; 1 ; 2$ 4 e 8 horas)

Tabela 11 - Análise do parâmetro de THMT

\begin{tabular}{|c|c|c|c|}
\hline & $\begin{array}{c}\text { Mo (hex) } \\
\left(\boldsymbol{\mu g ~ \mathrm { L } ^ { - 1 }}\right)\end{array}$ & $\begin{array}{c}\text { Mo (hex) + pol } \\
\left(\boldsymbol{\mu g ~ \mathrm { L } ^ { - 1 }}\right)\end{array}$ & $\begin{array}{c}\text { PAC } \\
\left(\boldsymbol{\mu g ~ \mathrm { L } ^ { - 1 }}\right)\end{array}$ \\
\hline C/F/Filtro - 0,5 h & 13,89 & 22,79 & 23,77 \\
\hline C/F/Filtro - 1 h & 21,74 & 23,91 & 23,87 \\
\hline C/F/Filtro - 2 h & 23,02 & 24,59 & 23,92 \\
\hline C/F/Filtro - 4 h & 30,14 & 24,72 & 24,00 \\
\hline C/F/Filtro - 8 h & 30,83 & 28,20 & 25,28 \\
\hline
\end{tabular}


Com base nos dados apresentados na Tabela 11 pode-se constatar que a água que passou pelo processo combinado de $\mathrm{C} / \mathrm{F} / \mathrm{Filtro}$, utilizando os três coagulantes testados, apresentou o mesmo comportamento, ou seja, um aumento expressivo na concentração de THMT após os primeiros 30 minutos de operação, seguido por um leve crescimento nos valores de THMT para tempos de retenção superiores. Mesmo apresentando esse comportamento de aumento da concentração de THMT com o aumento de retenção, os valores encontrados para o tempo de retenção máximo testado ficaram dentro dos limites da Portaria $\mathrm{n}^{\circ}$ 2914/2011 de $100 \mu \mathrm{g} . \mathrm{L}^{-1}$.

\section{CONCLUSÕES}

Pode-se concluir que o processo de C/F utilizando o coagulante Mo (hex) e Mo (hex) + pol com posterior filtração em carvão reduziu significativamente os parâmetros físicoquímicos analisados, produzindo água potável atendendo os limites da Portaria nº 2914/2011.

Assim, a Mo pode se apresentar como um coagulante alternativo eficiente para tratamento de água de abastecimento com reduzida formação de THM em concentrações muito abaixo do exigido pela portaria do ministério da saúde.

\section{REFERÊNCIAS}

AMERICAN PUBLIC HEALTH ASSOCIATION (APHA), 1995, Standard Methods for the Examination for Water and Wastewater. 19th ed., Washington.

BRASIL. Ministério da Saúde. Secretaria de Vigilância em Saúde. Diretriz nacional do plano de amostragem da vigilância ambiental em saúde relacionada à qualidade da água para consumo humano. Brasília: Secretaria de Vigilância em Saúde, 60 p, 2006.

DRINAN, J. E.; SPELLMAN, F. Water and Wastewater Treatment: A Guide for the Nonengineering Professional, Second Edition. Taylor \& Francis, 2012.

HONG, H. C., LIANG, Y., HAN, B. P., MAZUMDER, A., WONG, M. H., Modeling of trihalomethane (THM) formation via chlorination of the water from Dongjiang River (source water for Hong Kong's drinking water), Science of the Total Environment, v. 385, pp. 48 - 54. 2007.

KALIBBALA, H. M.; WAHLBERG, O.; HAWUMBA, T. J., 2009, - The impact of Moringa oleifera as a coagulant aid on the removal of trihalomethane (THM) precursors and iron from drinking waterl, Water Science and Technology, v.9, n.6, pp. 707-714.

WANG, Xiujuan et al. Synergistic effect of the sequential use of UV irradiation and chlorine to disinfect reclaimed water. Water research, v. 46, n. 4, p. 1225-1232, 2012. 